\title{
Improving the precision of intranasal oxytocin research
}

Adriano Winterton ${ }^{\mathrm{a}}$, Lars T. Westlye ${ }^{\mathrm{a}, \mathrm{b}}$, Nils Eiel Steen ${ }^{\mathrm{a}}$, Ole A. Andreassen ${ }^{\mathrm{a}}$, and Daniel S. Quintana ${ }^{\mathrm{a}}$

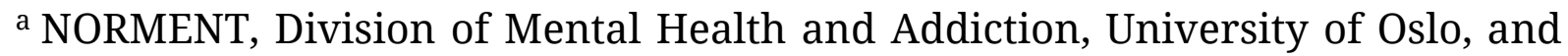
Oslo University Hospital, Oslo, Norway

${ }^{\mathrm{b}}$ Department of Psychology, University of Oslo, Oslo, Norway

Corresponding author: Daniel S. Quintana, NORMENT, KG Jebsen Centre for Psychosis Research, Building 49, Oslo University Hospital, Ullevål, Kirkeveien 166, PO Box 4956 Nydalen, N- 0424 Oslo, Norway, Ph: +47 2302 7350, fax: +47 2302 7333, email: daniel.quintana@medisin.uio.no

Word count: 4585 words

Keywords: oxytocin, social behaviour, neuroendocrinology, statistics 


\begin{abstract}
The neuropeptide oxytocin has been popularized for its role in social behaviour and nominated as a candidate treatment for several psychiatric illnesses. However, results so far have failed to reliably translate from animal models to human research. There have been justified calls to improve intranasal oxytocin delivery methodology, in terms of verifying that intranasal administration increases central levels of oxytocin. Nonetheless, improved methodology needs to be coupled with a robust theory of the role of oxytocin in behaviour and physiology to ask meaningful research questions. Moreover, stringent methodology based on robust theory may yield interesting results, but such findings will have limited utility if they are not reproducible. In this article, we outline how the precision of intranasal oxytocin research can be improved by the complementary consideration of methodology, theory, and reproducibility.
\end{abstract}




\section{Introduction}

The neuropeptide oxytocin has been popularised for its contributions to social behaviour and cognition ${ }^{1}$, with scholars pursuing intranasally administered oxytocin as a candidate therapy to address several psychiatric conditions, such as autism ${ }^{2}$ and anxiety ${ }^{3}$. Promising preclinical results demonstrating the association between oxytocin system dysfunction and social behaviour ${ }^{4,5}$, coupled with the recognition of the long-known roles of oxytocin in the maternal milk let-down reflex ${ }^{6}$ and childbirth ${ }^{7}$, has led to the characterization of oxytocin as a pro-social hormone ${ }^{8}$. However, experimental outcomes have not lived up to these expectations, with subsequent research emerging that the effects of oxytocin are exclusive to pro-social behaviours ${ }^{9}$ or have no significant effects on some facets of social behaviour at all ${ }^{10}$.

A "hype cycle" pattern can be observed with virtually any new concept, with maturing concepts eventually reaching a so-called "plateau of productivity” whereby performance matches expectations ${ }^{11}$. The intranasal oxytocin hype cycle pattern was recently described, concluding that the field is currently in a period of reorganisation ${ }^{12}$. While there have been thoughtful calls to improve our understanding of intranasal administration mechanisms to increase central oxytocin concentrations ${ }^{13}$, the impact of such efforts will be limited if researchers are asking the wrong questions of oxytocin (e.g., hypothesising that oxytocin is exclusively a pro-social hormone). The highly 
conserved nature of oxytocin signalling over 600 million years of evolution points to its instrumental role in organism survival ${ }^{14,15}$ (Box 1), yet its precise role is unclear. Thus, to better recalibrate theories it is crucial to better understand the purpose of oxytocin. In other words, how and why did oxytocin evolve? However, even if studies based on robust theories and stringent methods yield exciting results, it is critical that these outcomes are reproducible. For instance, several of the initial promising clinical results of intranasal oxytocin have been difficult to replicate. In this article, we provide a summary of current research efforts in and offer suggestions for ongoing research. We argue that by complementarily prioritising strong theory, methods and reproducibility, the therapeutic potential of targeting the oxytocin system will be more quickly realised. We also provide a summary of current research efforts in these areas and offer suggestions for ongoing research.

\section{Improving theory: What is the purpose of oxytocin signalling?}

While there have been many calls for the improvement of methods and research practices to increase the reproducibility of intranasal oxytocin research (e.g., $\left.{ }^{13,16}\right)$, there has been comparatively little consideration for the role of theory in reproducibility ${ }^{17}$. Broad theoretical frameworks can help connect a broad body of work across disciplines and can provide explanations beyond observed results. For instance, the role of oxytocin in physiological regulation can be used 
to inform its purpose in regulatory behaviour and cognition. Evaluating existing theories in light of current evidence is a useful exercise to determine their robustness and applicability across domains. To provide some historical context, the parturition and milk let-down effects of oxytocin have been known for over a century ${ }^{6,7}$, but it was not until the 1970 s that scholars first proposed that oxytocin may be involved in mammalian behaviour ${ }^{18}$. In humans, oxytocin was first thought to modulate pro-social cognition and behaviour, in light of the early results suggesting that intranasally administered oxytocin increases trust $^{19}$, however, the proposal that oxytocin increases trust has been challenged more recently ${ }^{20}$. Moreover, some effects of oxytocin appear to be sexually dimorphic (Box 2). Subsequent research emerged indicating that oxytocin also modulates non-prosocial behaviour (e.g., ${ }^{9}$ ), leading to the two prevailing theories in the field: that oxytocin increases the salience of social cues $^{21}$ and that oxytocin increases approach-related social behaviours ${ }^{22}$. In short, these theories propose that oxytocin modulates the perception and response to social cues, both positive and negative. However, these theories are inconsistent with more recent work suggesting that oxytocin also influences the processing of nonsocial stimuli as well as feeding-related behaviours. Moreover, they do not contain a strong account of how the manifold peripheral effects of oxytocin (e.g., metabolism) can influence thoughts and behaviours. Improving theory underlying the purpose of oxytocin by considering a wide of contexts, beyond 
its popularised role in social behaviour, can accelerate the translation of oxytocin research by providing more informed predictions for future research $^{23}$.

\subsection{The role of oxytocin beyond social cognition and behaviour}

\subsubsection{The influence of oxytocin on non-social cognition and behaviour}

A growing literature indicates that oxytocin is not exclusive to social effects ${ }^{24}$. For instance, recent work has demonstrated that the oxytocin-mediated pupil response to new stimuli is similar between social and non-social stimuli ${ }^{25}$. Intranasally administered oxytocin has also been found to increase the avoidance of emotionally-evocative social and non-social stimuli ${ }^{26}$ and to regulate discrimination learning related to non-social cues in rats ${ }^{27}$. Human work from Singer and colleagues found that in participants receiving painful hand stimulation, intranasal oxytocin administration reduced amygdala activation $^{28}$. In another human fMRI study by Zhao and colleagues found that use of an intranasal oxytocin spray increased intrinsic connectivity in ventral caudal-dorsal anterior cingulate cortex pathways engaged in reward and salience processing and pallidal-frontal motor pathways involved in action initiation and approach ${ }^{29}$. In sum, emerging research is pointing to the idea that oxytocin may not exclusively moderate the perception and response to social stimuli. Instead, oxytocin seems to influence the perception of stimuli that are 
personally relevant ${ }^{24}$, which are typically social in nature, but can also encompass non-social stimuli.

\subsubsection{Feeding behaviour and energy balance.}

Emerging preclinical evidence in both animals and humans indicates that oxytocin is associated with feeding behaviour and energy balance. This link is supported by neuroanatomy (Fig. 2). Oxytocin axonal projections from parvocellular neurons innervate the dorsal vagal complex (DVC), where neurons express oxytocin receptors ${ }^{30}$. These oxytocin axonal projections, along with others to the spinal cord, parabrachial nuclei, periaqueductal grey, ventrolateral medulla, and sympathetic paraganglionic neurons in the spinal $\operatorname{cord}^{31}$, regulate parasympathetic and sympathetic reflexes that coordinate the digestive functions of the stomach and oesophagus. Neurons from the nucleus of the solitary tract project back to the PVN and other areas of the hypothalamus to provide feedback for feeding behaviour and are important for meal termination. Thus, modulation of the dorsal vagal complex by the PVN, including by oxytocin neurons, is tied to both gastric motility and feeding behaviour ${ }^{32}$. Oxytocin has also been experimentally linked to feeding behaviours in primates and rodents, inhibiting food intake in a dose-dependent fashion $^{33}$. Oxytocin may reduce food intake by strengthening inhibitory action on cholecystokinin modulating leptin and by its impact on peripheral satiety 
signalling on central receptors ${ }^{34}$. Two preliminary studies in humans report that intranasal oxytocin administration decreases food intake and improves insulin sensitivity in healthy men ${ }^{35,36}$, and prolonged treatment with intranasal oxytocin reportedly reverses pre-diabetic changes ${ }^{37}$.

Oxytocin administration was shown to be protective against high fat dietinduced obesity in rodents and rhesus monkeys ${ }^{38}$, while oxytocin knockout mice prefer sucrose and develop pre-diabetic changes independent of food intake ${ }^{39}$. The anti-obesity effects have been shown to last for weeks even after cessation and washout of oxytocin treatment in animals ${ }^{38,40}$, which supports the proposed feed-forward mechanism of central oxytocin ${ }^{41,42}$. Studies on the oxytocin system point to its modulatory effects on caloric intake and body weight, with exogenous oxytocin has been shown to reduce caloric intake in humans ${ }^{36}$ as well as body weight in dietarily-induced-obese rhesus monkeys through decreased food intake as well as increased energy expenditure and lipolysis ${ }^{43}$.

2.2.3. Deciphering the role of oxytocin by mapping receptor locations in the brain Identifying the location of oxytocin receptors in the brain is a crucial step for developing theories of the effects of oxytocin and constraining the range of mental states that oxytocin signalling is likely to influence. Moreover, without a good understanding of oxytocin receptor locations, it is difficult to determine whether non-significant results are due to a lack of target engagement, or an 
inefficacious treatment ${ }^{44}$. Animal research has located oxytocin receptor expression in the ventral pallidum, olfactory bulb, amygdala, dorsal vagal nucleus, and hypothalamus, with these patterns believed to reflect the social organisation of a species (e.g. social monogamy) ${ }^{4}$ as well as individual differences in social behaviour ${ }^{45}$. Animal research has also demonstrated that experimentally increasing oxytocin receptor expression in specific brain regions in the prairie vole can influence their behaviour ${ }^{46}$. While the location and role of oxytocin receptors in non-human mammals is becoming wellcharacterised $^{4,15}$, the distribution and functional neuroanatomy of oxytocin receptors in humans has historically been poorly understood. Prior research has used post-mortem brain samples from a limited number of brain regions ${ }^{47,48}$, but this approach limits conclusions regarding the role of the location of these receptors in physiology and behaviour, as mental states and physiological processes are supported by neural networks comprising several rather than single brain regions.

An indirect means of simultaneously assessing the role of oxytocin in various mental states is to identify the location of oxytocin receptors across the brain and then match these patterns against networks known to support various mental states according to fMRI data. A recent study investigated which mental state brain activation patterns were most strongly associated with voxel-byvoxel oxytocin receptor expression patterns generated from the Allen Human 
Brain Atlas (Fig. 1). By combining this data with the NeuroSynth fMRI metaanalysis tool ${ }^{49}$, this study revealed that oxytocin receptors are expressed in areas which underlie mental states broadly categorised as anticipatory, appetitive, and aversive ${ }^{50}$, consistent with recent regional cerebral blood flow data from Paloyelis and colleagues ${ }^{51}$. Of interest, the data pointed to expression of the oxytocin receptor in the olfactory region of the brain, which appears to be the first brain region to come in contact with intranasally administered oxytocin, as mentioned above ${ }^{52}$. As expected, there was a relationship between oxytocin receptor gene expression patterns and "emotional" mental state activity patterns, but this analysis also pointed to the important role of the oxytocin system in reward and learning, consistent with suggestions that dopamine plays an important role in oxytocinergic signalling ${ }^{53}$. Related to this, similar spatial distributions were found between oxytocin receptor expression and the expression of dopamine receptor genes DRD2 and DRD5. Another intriguing result was the apparent role of oxytocin signalling system in metabolic regulation, as the "taste" mental state activity pattern was highly correlated with oxytocin receptor expression. Moreover, an exploratory analysis of the strongest relationship among all other 20,737 protein-coding genes and the oxytocin receptor revealed that three genes that regulate energy and metabolism (NTSR2, GLUD1, GLUD2) were among the top five expressed genes with the strongest similarities in the neuroanatomical distribution. 


\subsubsection{The effects of oxytocin beyond the central nervous system}

Superficially, it would seem that the role of oxytocin outside the central nervous system would have little relevance for understanding its cognitive and behavioural roles. However, as the physiological effects of oxytocin likely evolved first, with complex behavioural effects evolving later ${ }^{54}$, it would be unusual if oxytocin-mediated changes in physiology had no effect on mental states (Box 1). The physiology of the oxytocin system can provide clues as to its purpose and role. Oxytocin is almost exclusively produced within hypothalamic neurons, and secreted both centrally and peripherally. Oxytocin is secreted to the peripheral circulation via the neurohypophysis, and oxytocin receptors are expressed in a wide number of different organs and tissue types, such as the heart, kidneys, muscle tissue, and gastrointestinal tract ${ }^{55}$. Adding to this complexity, bone marrow cells also synthesize OXT, suggesting the existence of autocrine and paracrine interactions ${ }^{56,57}$. The diverse location of peripheral oxytocin receptors and sites of production provides evidence for its varied roles in physiological processes.

Oxytocin neuron projections in the CNS suggest that the varied functions of oxytocin are not limited to behaviour, with axonal projections from the Paraventricular Nucleus (PVN) and Supraoptic Nucleus (SON) to various areas involved in the regulation of energy balance and metabolism ${ }^{32}$. This is coupled 
with the dendritic release of oxytocin to structures in the proximity of the PVN and SON that increase the complexity of the dynamics of oxytocin release. Dendritically released oxytocin acts within the PVN and SON, and affects the oxytocin neurons themselves. It is probably also the main source of oxytocin signalling to two relatively close sites that express abundant oxytocin receptors: the amygdala, which contains only a few oxytocin-containing fibres, and the ventromedial nucleus (VMN), which appears to contain none. The VMN is important for glucose homeostasis ${ }^{58}$ and controls related feeding behaviour and energy expenditure, modulated by oxytocin at this site ${ }^{59}$.

Both oxytocin producing neurons and their receptors are found in the heart and large vessels ${ }^{60}$. The presence of both oxytocin and its receptors suggests autocrine/paracrine roles for the peptide. Oxytocin influences the peripheral cardiovascular system by binding either to oxytocin receptors in the heart, or to the vasculature to lower preload and contractility of the heart ${ }^{61,62}$. In rats, oxytocin has been shown to stimulate the release of atrial natriuretic peptide from the heart, which in turn regulates blood volume and pressure promoting sodium and water excretion ${ }^{63,64}$ and vasorelaxation of the coronary $\operatorname{arteries}^{65}$

The effects of oxytocin on the cardiovasculature are not limited to peripheral stimulation, but also to centrally-mediated regulation. Oxytocin neurons from the PVN project to vasomotor centres in the brainstem. In 
response to physiological challenges, oxytocin in the hypothalamus can change vascular tone through autonomic nerves and in turn modulate cardiovascular activity $^{62,66}$. The PVN projects directly to the sites that control cardiorespiratory function - the intermediolateral cell columns, phrenic motor nuclei in the spinal cord, rostral ventrolateral medulla, and the rostral nuclei in the ventral respiratory column in the brainstem, as well as the locus coeruleus and, as mentioned before, the dorsal vagal complex ${ }^{64,67}$. These are also involved in autonomic (sympathetic) responses to stress ${ }^{68}$. Animal and human experiments point towards a modulating link of central and peripheral oxytocin on physiological reactions to stress, affecting catecholaminergic release and the hypothalamus-hypophysis-adrenal axis ${ }^{69,70}$. However, activation of the Hypothalamic-Pituitary-Adrenal (HPA) axis due to stress can alter the functioning of the suprachiasmatic nucleus, decreasing the synthesis and release of endogenous oxytocin ${ }^{71}$.

Although several peripheral effects of oxytocin have been described in the literature, including bone mass regulation ${ }^{57}$, such effects are not thought to directly influence our thoughts and behaviours. In contrast, the cardiac autonomic system, which has been associated with oxytocin signalling ${ }^{72,73}$, has been linked with social behaviour and cognition ${ }^{74}$. Parasympathetic nervous system activity in particular can be non-invasively measured using heart rate variability (HRV), with lower HRV associated with reduced parasympathetic 
nervous system activity ${ }^{75}$. The Polyvagal Theory suggests that the mammalian cardiac autonomic system has evolved to support social behaviour ${ }^{74}$, with increases in parasympathetic nervous system activity promoting both the perception ${ }^{76}$ and projection ${ }^{77}$ of social behaviour (but see ${ }^{78}$ ). Research has shown that intranasal oxytocin increases $\mathrm{HRV}^{79}$, suggesting that oxytocin increases parasympathetic activity, which may partly explain its reported peripheral effects. It is difficult to determine whether an increase in HRV is primarily a central or peripherally mediated effect. But given that intranasal, but not intravenous, oxytocin administration influences the perception of social cues ${ }^{80}$, these effects are probably centrally mediated via the central autonomic network ${ }^{81}$.

\subsection{Improving theory can accelerate research translation}

Altogether, evidence suggests that the oxytocin system has a diverse set of effects on cognition, behaviour, and physiology. Prevailing theories of oxytocin primarily consider its social aspects, with little consideration of the non-social aspects or how oxytocin influences behaviour and physiology. Narrow approaches can limit hypothesis testing and the applicability of findings to different contexts, such as non-social behaviours and physiology. It has been recently proposed that the varied effects of oxytocin are in fact a coordinated effort to support allostasis ${ }^{82}$, which is the process of maintaining stability in 
changing environments. This theory, which was derived from both an evolutionary and mechanistic perspective, accounts for the function of oxytocin over a range of contexts and is applicable in both animal and human models.

\section{Improving methods: How can researchers enhance intranasal oxytocin} administration to the brain?

While strong theory can help ask better questions, this is of little consequence with weak methodology. An especially pertinent question in terms of methodology for the present discussion is whether researchers can reliably manipulate oxytocin levels in the brain. As intracerebral administration is impractical in humans and the blood brain barrier is thought to limit intravenously administered oxytocin reaching the central compartment, researchers have turned to intranasal administration to manipulate oxytocin levels in the brain. This approach has been applied under the assumption that intranasally administered oxytocin increases oxytocin levels in the central nervous system via nose-to-brain pathways, exerting its psychological and behavioural effects via the occupation of central oxytocin receptors. These premises have historically only been substantiated by indirect evidence and been the subject of strong criticism ${ }^{13}$, but more recent research has begun to address these critiques. 


\subsection{Evidence for nose-to-brain transport}

Intranasally administered molecules deposited on rodent olfactory and respiratory epithelia can be transported into the brain via olfactory and trigeminal nerve fibres ${ }^{83,84}$. These epithelia are located in the hard-to-access upper and posterior regions of the nasal cavity, a deposition target that is not well-suited for traditional pump-actuated nasal sprays ${ }^{85}$. Other areas of the nasal cavity that are closer to the nostrils are heavily vascularised ${ }^{86}$. Oxytocin spray deposition on these vascularised regions can facilitate entry into the bloodstream. But given the relatively large size of oxytocin molecules (1007 daltons), it is believed that very little of this circulating oxytocin crosses the blood brain barrier $^{13}$. Smith and colleagues provide compelling evidence that intranasally administered oxytocin can enter the brain in mice at behaviourally relevant concentrations ${ }^{87}$. Research also indicates that intranasal oxytocin administration increases levels of oxytocin in cerebrospinal fluid in both humans $^{88}$ and non-human primates ${ }^{89,90}$. Importantly, intranasal administration increases oxytocin levels in brain regions in mice that are critical for social behaviour and rich in oxytocin receptors ${ }^{87}$.

It is plausible that intranasally administered oxytocin, which elevates circulating plasma levels of oxytocin ${ }^{80,88-90}$, crosses the blood brain barrier to exert its effects. Indeed, the first reported investigation of the effect of oxytocin on psychiatric symptoms used intravenous delivery in participants diagnosed 
with autism ${ }^{91,92}$. A single administration of intravenous oxytocin reduced repetitive behaviours and improved the retention of social information, suggesting that intravenously administered oxytocin can modulate behaviour and cognition, presumably via central oxytocin activity.

Additional pharmacokinetic experiments in oxytocin (OXT) geneknockout mice in which oxytocin was delivered intraperitoneally and intranasally, produced increases in plasma and CSF levels of oxytocin ${ }^{87}$ as well as replicating previous findings ${ }^{93}$. As these results were found in $O X T$-knockout mice, who cannot produce oxytocin, increased central levels could be solely attributed to exogenous administration. Relatedly, the administration of both intranasal and intravenous oxytocin in rhesus macaques increased CSF oxytocin, but only intranasally administered oxytocin led to the central release of endogenous oxytocin ${ }^{90}$.

To determine the route of intranasally administered oxytocin into the human brain, research has compared the effects of intranasal and intravenous oxytocin delivery on social cognition, brain activity, and physiology, in neurotypical adult males ${ }^{80,94,95}$. While intranasal oxytocin was found to modulate the processing of social cues $^{80}$, decrease amygdala activity during the processing of social cues ${ }^{94}$, and to modulate pupil diameter ${ }^{95}$, no such effects were found after intravenous oxytocin, despite comparable levels of circulating oxytocin ${ }^{80}$. Together, these results suggest that intranasally administered oxytocin 
primarily exerts its central effects via the nose-to-brain route, instead of crossing the blood brain barrier.

While these human studies have been informative, these approaches are still not a direct confirmation of the nose-to-brain oxytocin administration route, as intranasally administered oxytocin may enter the brain via a yet-to-be recognised route via the nasal cavity. A more direct approach to confirm direct transport via the olfactory and trigeminal nerve fibres to the brain is to use an oxytocin receptor radiotracer and then observe the destination of intranasally administered oxytocin using PET imaging. Recent animal research using a novel oxytocin receptor radiotracer demonstrated an increased detection of the radiotracer in the olfactory bulbs of rats after intranasal administration compared to intravenous administration, consistent with transport via nerve fibres $^{52}$. While this is an important first step, research is yet to demonstrate these effects in humans.

\subsection{Improving intranasal oxytocin methods can accelerate oxytocin}

\section{research translation}

A central premise of intranasal oxytocin research is that this delivery mode increases levels of oxytocin in the brain. A strong theoretical foundation for a research question is of little value if researchers cannot increase levels of oxytocin in the brain. As shown above, several lines of evidence suggest that 
intranasally delivered oxytocin can increase central levels in the brain. However, there are several factors that can hinder the intranasal administration of therapeutics targeted to the brain, such as intranasal delivery technique and nasal cavity physiology ${ }^{96}$. Following published guidelines for intranasal oxytocin administration ${ }^{85}$, can help ensure increased levels in the brain. The collection of CSF samples to confirm increased central levels is impractical for regular use, however, the collection of blood plasma samples after intranasal administration may provide an indirect proxy of central levels of oxytocin ${ }^{97}$.

\section{Improving reproducibility: How can we improve the reliability of}

\section{intranasal oxytocin results?}

Even if researchers manage to test precise hypotheses using valid methods for increasing oxytocin levels in the central compartment, this is of little translational value unless results are reproducible. A large part of the biobehavioural sciences are experiencing a so-called "reproducibility crisis"98, which has been attributed to a panoply of research practices. It is widely known that oxytocin studies have historically been statistically underpowered, with intranasal oxytocin RCTs reported to have $16 \%$ statistical power on average ${ }^{16}$. Thus, there is a need to calculate precise sample size estimates when planning studies (Box 3). Relatedly, the Registered Reports format ${ }^{99}$ has been implemented in several journals in an effort to increase the reproducibility of 
hypothesis-driven research and reduce publication bias. With this format, manuscripts are assessed in two stages. In the first stage, the rationale and methods are reviewed before data collection. If the reviewers and editors approve the manuscript at this stage, the manuscript is given in-principle acceptance, as long as the conclusions are justified by the results, which are presented at the second stage of review. While the Registered Report format is only available in a limited (but growing) selection of journals, there are two important research practices that are especially relevant for to help accelerate the translation of oxytocin research: using tools to assess evidence for a null hypothesis the publication of open data. The following sections will cover these practices, highlighting prior applications in oxytocin research.

\subsection{Evidence for a null hypothesis}

Emerging oxytocin theories need to be falsifiable as hypotheses cannot be rejected or refined without falsification ${ }^{100}$. However, with traditional frequentist inference, one cannot quantify the evidence in favour of a null hypothesis, only a failure to reject the null hypothesis ${ }^{101}$. Thus, if a study yields a non-significant $p$-value, then there are two possible conclusions: there was either support for the null hypothesis or the sample was not sensitive enough to detect a difference (i.e., the sample size was too small). It is not possible to provide a precise conclusion based on a non-significant $p$-value alone, no matter 
the size of the $p$-value. In practice, this means that most studies reporting nonsignificant results are not especially informative ${ }^{101}$. Such studies demonstrate a failure to reject the null hypothesis, but they do not provide information as to why this occurred. There are two emerging methods for calculating evidence for a null hypothesis or model: frequentist equivalence testing ${ }^{102,103}$ and Bayesian hypothesis testing ${ }^{104}$. These are by no means new methods, but only more recently have they become available in openly accessible point-and-click software packages (e.g., JASP and JAMOVI) and R packages.

Bayesian hypothesis testing provides the means to estimate the relative evidence for a null model against an alternative model, in the same manner as an odds ratio ${ }^{104}$, which are typically easy to interpret. Several tutorials are now available for Bayesian hypothesis testing using free point-and-click software (e.g., $\left.{ }^{104}\right)$, including one using oxytocin research as an example ${ }^{105}$. If one wishes to remain within a frequentist framework, then equivalence testing can be used to reject effects as large or larger than a smallest effect size of interest (Box 3) ${ }^{103}$. The application of equivalence testing on non-significant results has been revealing in the context of oxytocin research, as it has shown that in a sample of 34 non-significant effects from a lab’s "file draw", over 70\% of these results were due to insensitive study designs ${ }^{106}$, as these studies did not demonstrate statistical equivalence. Using the same approach for another set of oxytocin study data, Tabak and colleagues ${ }^{107}$ similarly reported that almost $60 \%$ of non- 
significant results were due to insensitive study designs. Altogether, sensitive study designs coupled with tools to determine evidence for null and/or equivalent effects can help accelerate the translation of intranasal oxytocin research by facilitating the refinement or rejection of hypotheses.

\subsection{Open data}

Among several recommendations for improving the intranasal oxytocin research from Leng and Ludwig ${ }^{13}$ was the promotion of data sharing. Not only does data sharing ensure the reproducibility of reported results, but it also facilitates data exploration for hypothesis generation. While there are few impediments for sharing raw data in animal research, this issue is more complex in human research due to the risk of identification, especially with sensitive data. One solution is the generation of synthetic datasets, which share the same statistical properties of the original dataset, however, none of the cases match the original dataset, which reduces the risk of re-identification to almost nil ${ }^{108}$. Synthetic datasets were originally developed for sharing confidential census data, but are also appropriate for sharing data in the biobehavioural sciences, including intranasal oxytocin research ${ }^{109}$. Sharing synthetic data will help ensure the reproducibility of reported results, while helping safeguard the privacy of study participants. 


\section{Conclusions}

Intranasal oxytocin research has arrived at a critical juncture. The field is now emerging from a period of disillusionment due to disappointing results ${ }^{12}$ and becoming aware of the limitations of currently used methods and study designs with a succession of critical articles ${ }^{13,20,110,111}$. But despite this information, it is not yet clear whether the field will act on these warnings or maintain the status quo. Moreover, even if the behavioural oxytocin field improves its theoretical and methodological approaches, resources might be squandered if results do not replicate. Thus, improving theory, methods, and reproducibility, as described in this article, will help to establish the necessary evidence for the role of oxytocin in cognition and in the treatment of mental disorders.

In this review, we have primarily covered research investigating one proximate question (how does oxytocin work?) and one ultimate question (what is its purpose?) to better understand oxytocin signalling, in an effort to improve study precision. However, these types of questions tend to cover how oxytocin signalling works at a given slice of time, instead of how a given phenotype develops over the course of time (see Box 4). As mental illnesses tend to emerge at distinct life stages ${ }^{12}$, understanding how oxytocin signalling changes across development can help clarify its function and better reveal its role in the aetiology of mental illnesses with strong neurodevelopmental aspects. Given the conservation of oxytocin signalling across evolutionary time (Box 1$)^{54}$, it is also 
likely to provide important survival value. Thus, a more comprehensive understanding of the evolution of the oxytocin system, from the ancient sea squirt ${ }^{113}$ to humans, will help better uncover the functional significance of oxytocin signalling 23 .

This review is by no means a final word on the state of intranasal oxytocin research. Here, three areas have been raised that require careful attention to improve the precision of oxytocin research-theory, methods, and reproducibility—but there are certainly more. By rejecting and refining hypotheses informed by well-developed theory, using cutting-edge methodology, and by using replicable research practices, scholars can improve our understanding of the oxytocin signalling system and uncover the potential of intranasal oxytocin as a psychiatric therapeutic. 


\section{Acknowledgements}

This work was supported by an Excellence Grant from the Novo Nordisk

Foundation (NNF160C0019856), the European Research Council under the

European Union's Horizon 2020 research and Innovation program (ERC StG, Grant 802998). 


\section{Competing interests}

The authors declare no competing interests. 


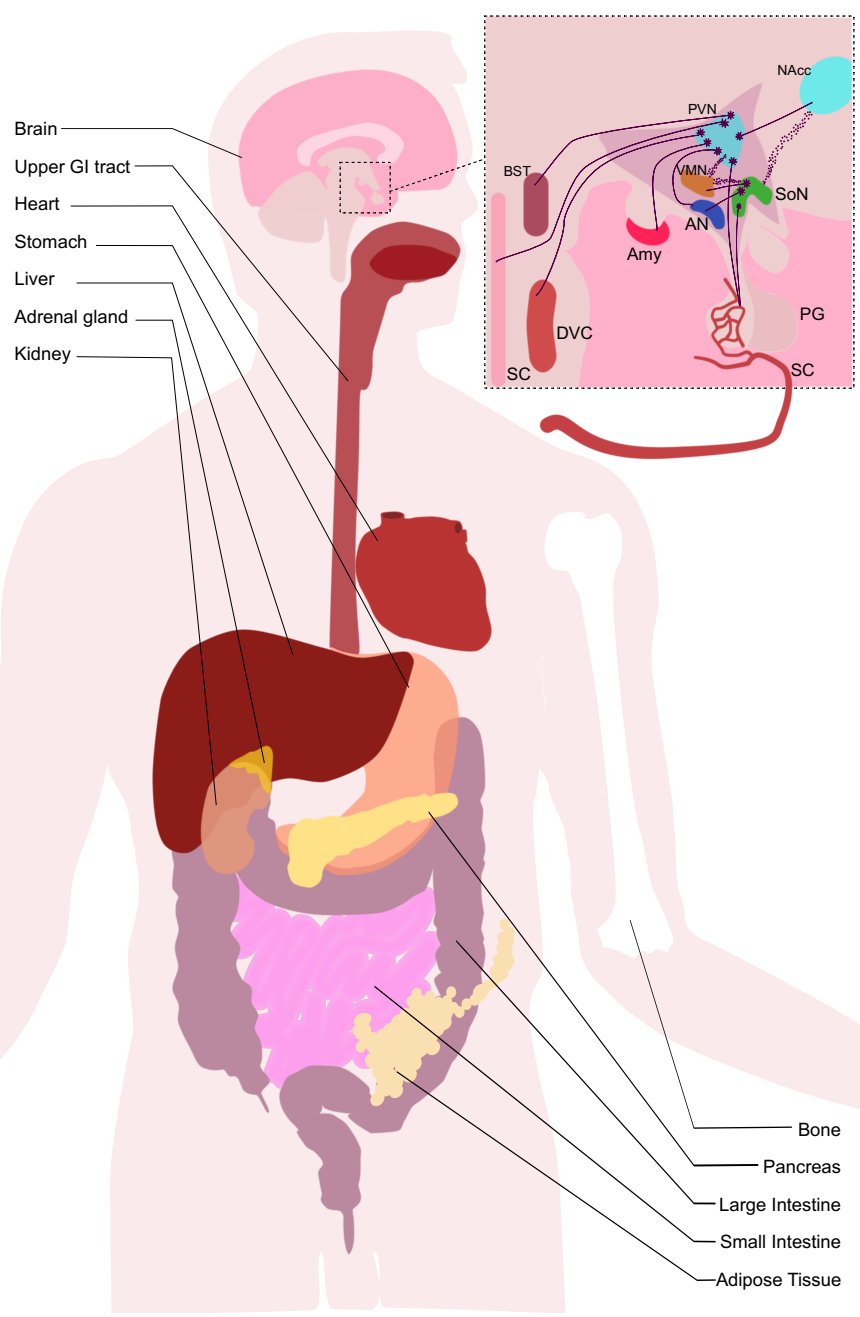

Figure 1. Oxytocin production and targets. Projections from parvocellular neurons in the Paraventricular Nucleus (PVN) innervate the dorsal vagal complex $(D V C)$, parabrachial nuclei, periaqueductal grey, ventrolateral medulla, and the spinal cord (regulating digestive functions of the oesophagus and stomach ${ }^{30,31}$, thermogenesis, pain processing and erectile function ${ }^{32}$ ). Through the neurohypophysis, oxytocin is released into systemic circulation where it affects a wide number of different organs and tissue types that express the oxytocin receptor, such as the heart, kidneys, pancreas, liver, muscle tissue, adipose tissue, and gastrointestinal tract ${ }^{55,115}$. Oxytocin can also modulate vascular tone and cardiovascular activity through autonomic nerves ${ }^{62,66}$. Oxytocin is also thought to regulate bone mass ${ }^{57}$, and bone marrow cells also synthesize OXT, suggesting its function as a local messenger ${ }^{56,57}$. 


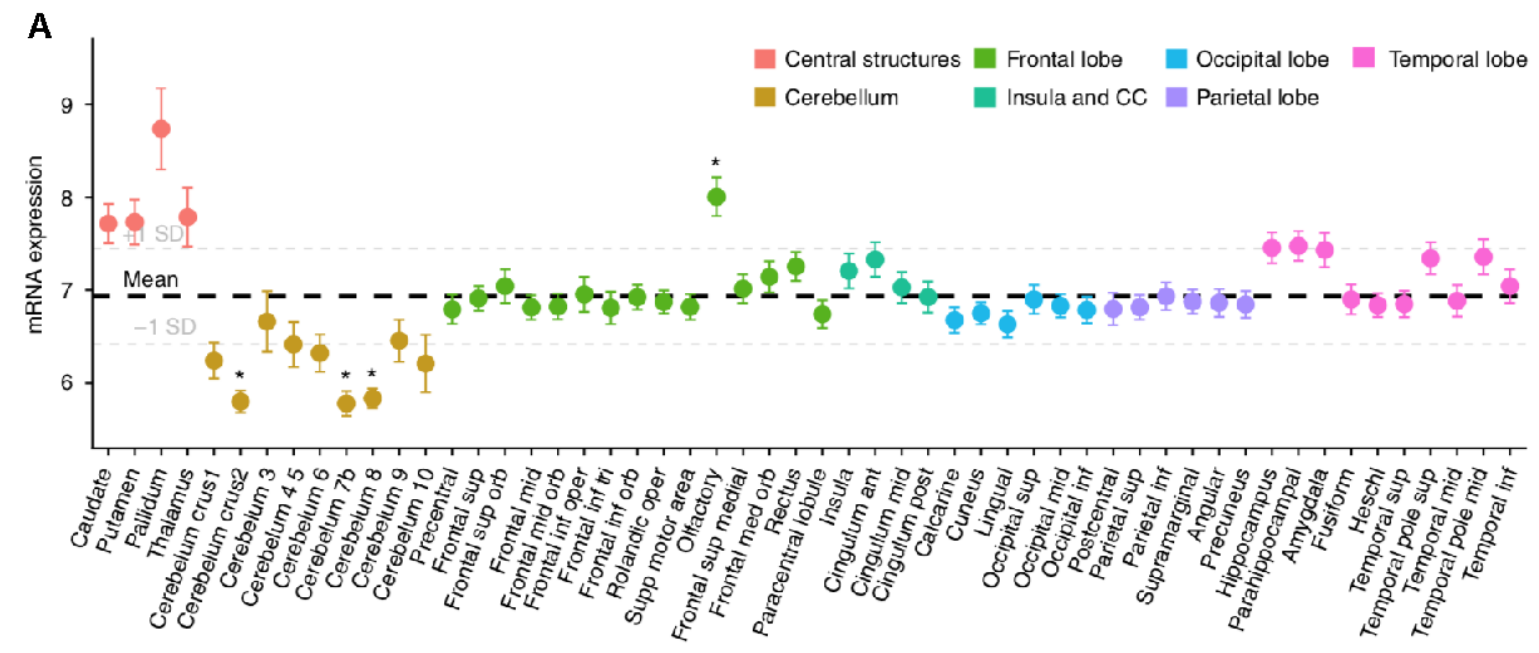

B

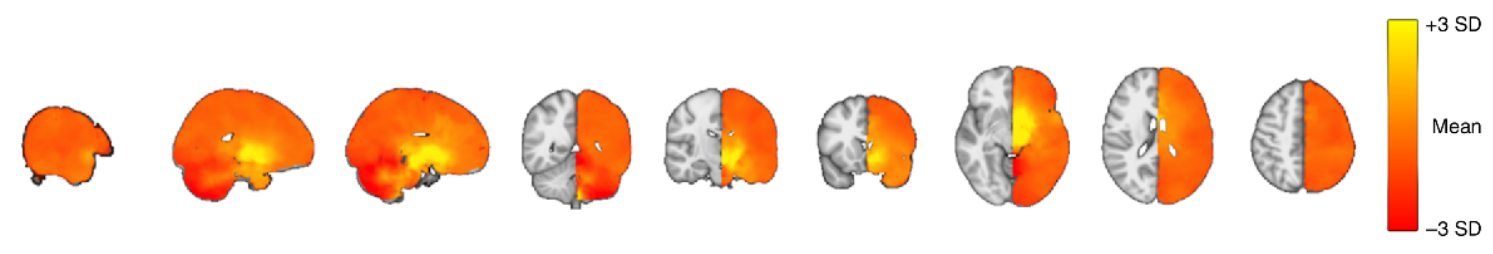

Figure 2. Expression of the oxytocin receptor in the human brain. Oxytocin receptors are highly expressed in central regions of the brain. Expression levels are shown in terms of brain regions from Automated Anatomic Atlas brain regions (A) and on a voxel-by-voxel level across the brain (B). Figure adapted from Quintana et al ${ }^{50}$. 


\section{Box 1. The evolutionary journey of the oxytocin signalling system}

Better understanding the ontogeny, phylogeny and adaptive significance of a signalling pathway can help explain what makes it vulnerable to malfunction ${ }^{116}$. Oxytocin-like peptides first appeared before vertebrates branched off from invertebrates ${ }^{54}$. The most ancient physiological action of oxytocin-like peptides is myoactivity-the stimulation of tissue contraction. Myoactive effects of oxytocin and oxytocin-like peptides can be observed in rhythmic-patterned behaviours in earthworms (Eisenia foetida) ${ }^{117}$, leeches (Withmania pigra) ${ }^{117}$ and sea squirts (Ciona intestinalis) ${ }^{118}$, among other species. The effects of oxytocin signalling on smooth muscle contraction persist in humans, where oxytocinmediated smooth muscle contraction is involved in reproductive mechanisms such as copulation, childbirth and lactation as well as gastric emptying and cardiovascular and osmotic regulation ${ }^{119}$. The different species-specific functions of oxytocin-like systems lends support to evolutionary perspectives that theorise how a broad range of complex adaptive systems arise out of coopting mechanisms from basic ones ${ }^{54,119}$.

The progression from stereotyped responses to more complex behavioural functions is still unclear. Learning could arguably be the earliest link beyond reproduction. This has been demonstrated in primitive invertebrate organisms such the roundworm (Caenorhabditis elegans) where an oxytocin-like systems is used to regulate gustatory associative learning ${ }^{120}$. Effects on learning are present in vertebrates too, with oxytocin improving memory in a variety of tasks ${ }^{121}$.

Oxytocinergic projections from the hypothalamus to the pituitary highly conserved across placental mammals ${ }^{122}$. However, there are wide variations in central oxytocin receptor distribution, particularly in limbic areas ${ }^{123}$. This suggests an ancient role of oxytocin signalling for the regulation of physiological functions, while the variations in central oxytocin receptor patterns likely reflect the impact of different environmental pressures on a species social organization $^{119}$. 


\section{Box 2. Sex Differences in Oxytocin signalling}

Oxytocin is involved in sex-specific reproductive functions ${ }^{6,7}$ but little is known about non-reproductive sex-specific functions. Most human intranasal oxytocin studies have been conducted solely in males (or with a heavy bias), or did not factor in sex differences in their analyses ${ }^{124}$. With regards to OXTR expression in the brain, a recent study has shown a lack of evidence for strong sex differences ${ }^{50}$; however, more studies have reported diverging or even opposite effects of intranasal administration of oxytocin ${ }^{124}$. More evidence for sexspecific differences in the oxytocin system come from studies finding differences in plasma oxytocin levels ${ }^{125,126}$ as well as sex-specific behavioural correlations with oxytocin ${ }^{127}$. It must be noted, however, that there is a great need for improvement and standardisation in oxytocin measurements ${ }^{128}$. Numerous further studies have also demonstrated sex differences in neural responses to intranasal oxytocin administration ${ }^{129-131}$. This suggests that these sex differences may be mediated by the moderating impact of sex steroids. Other studies have found no sex differences in the effects of oxytocin in other domains $^{132,133}$, pointing to the fact that some, but not all, effects of oxytocin exhibit sex differences, in line with current proposed models of the interaction between sex steroids and oxytocin ${ }^{134}$, that could help explain localised sexspecific interactions between the two ${ }^{135}$. Future studies should, if possible, ensure that they account for sex in the main effects of their analyses and any clinical translation of intranasal oxytocin should carefully account for potential sex differences. 


\section{Box 3. Sample size estimation and the smallest effect size of interest}

There are two primary limiting factors for researchers when performing power analysis. First, determining an effect size of interest can be difficult, as pilot studies provide little guidance and powering studies based on past results is inadvisable due to effect size inflation via publication bias ${ }^{136}$. Rather than using rule-of-thumb effect sizes (e.g., Cohen's small/medium/large conventions) for the design (and critique) of oxytocin research, there has been an emerging push for scholars to carefully consider the smallest effect size of interest (i.e., the effect size that is the most minimally-interesting) for their study design and statistical test, particularly in the medical sciences ${ }^{137}$. Calculating a smallest effect size of interest can help rule out statistically equivalent results, but also help ensure the detection of effects that are meaningful. There are no rules-of-thumb that can help determine a smallest effect size of interest for a given study design, but some suggestions for how to consider this issue have been proposed ${ }^{138}$. Second, while power analysis is available using free tools, such as $\mathrm{G}^{*}$ power $^{139}$ and various $\mathrm{R}$ packages, these packages are limited to relatively straightforward research designs, such as correlations, t-tests, and one-way ANOVAs. Data simulation tools can be used for the power analysis of more complex mixed model research designs ${ }^{140}$. 


\section{Box 4. The developmental role of oxytocin}

The development of oxytocin neuron occurs in the early phases of embryogenesis and is highly conserved in several mammalian and nonmammalian species ${ }^{141}$. Oxytocin synthesis in the human brain begins before birth $^{142}$, suggesting that it is involved in foetal physiology and development. In humans the SON and PVN are completely formed at 25-weeks gestation and the number of oxytocin neurons is fairly similar from gestation to adulthood, while oxytocin receptor expression levels in the brain vary across the lifespan ${ }^{141}$. Oxytocin receptor expression in the brain changes across mammalian development, exhibiting species-specific ${ }^{143}$ oxytocin receptor expression patterns during different developmental periods ${ }^{141,144}$, pointing to speciesspecific survival challenges ${ }^{119}$.

Oxytocin is involved in cortical development following to sensory experiences in neonatal non-human mammals ${ }^{145}$, with dysfunction in this system impairing neonatal social learning in rodents ${ }^{146,147}$. Gene regulatory features of oxytocin neuronal cells point to dual sensory-neurosecretory character, suggesting involvement in the conversion of sensory inputs into behavioural responses ${ }^{54}$. In humans, social and stress-related behaviours tied to oxytocin (such as soothability and sociability) are first detected during toddlerhood ${ }^{148,149}$.

Evidence overall suggests that oxytocin signalling problems during early life hinders later affiliative behaviour due to a disruption of learning processes ${ }^{119}$. The oxytocin signalling system in mammals therefore seems to play an instrumental role in sensory learning, for both social and non-social cues, in the developing brain ${ }^{143}$. The plasticity of the oxytocin system for the facilitation of learning may leave the oxytocin system susceptible to early life stressors. increasing the potential for the development of psychiatric disorders ${ }^{150}$. 


\section{References}

1. Guastella, A. J., Graustella, A. J. \& MacLeod, C. A critical review of the influence of oxytocin nasal spray on social cognition in humans: evidence and future directions. Horm. Behav. 61, 410-418 (2012).

2. Yatawara, C. J., Einfeld, S. L., Hickie, I. B., Davenport, T. A. \& Guastella, A. J. The effect of oxytocin nasal spray on social interaction deficits observed in young children with autism: a randomized clinical crossover trial. Mol. Psychiatry 21, 1225-1231 (2016).

3. Guastella, A. J., Howard, A. L., Dadds, M. R., Mitchell, P. \& Carson, D. S. A randomized controlled trial of intranasal oxytocin as an adjunct to exposure therapy for social anxiety disorder. Psychoneuroendocrinology 34, 917-923 (2009).

4. Insel, T. R. \& Shapiro, L. E. Oxytocin receptor distribution reflects social organization in monogamous and polygamous voles. Proc. Natl. Acad. Sci. 89, 5981-5985 (1992).

5. Olazabal, D. E. \& Young, L. J. Oxytocin receptors in the nucleus accumbens facilitate "spontaneous" maternal behavior in adult female prairie voles. Neuroscience 141, 559-568 (2006).

6. Schafer, E. A. \& Mackenzie, K. The action of animal extracts on milk secretion. Proc R Soc Lond B 84, 16-22 (1911).

7. Dale, H. H. On some physiological actions of ergot. J. Physiol. 34, 163-206 (1906). 
8. Coghlan, A. 'Cuddle chemical'eases symptoms of schizophrenia. New Sci. 207, 10 (2010).

9. Shamay-Tsoory, S. G. et al. Intranasal Administration of Oxytocin Increases Envy and Schadenfreude (Gloating). Biol. Psychiatry 66, 864-870 (2009).

10. Dadds, M. R. et al. Nasal oxytocin for social deficits in childhood autism: A randomized controlled trial. J. Autism Dev. Disord. 44, 521-531 (2014).

11. O'Leary, D. E. Gartner's hype cycle and information system research issues. Int. J. Account. Inf. Syst. 9, 240-252 (2008).

12. Alvares, G. A., Quintana, D. S. \& Whitehouse, A. J. O. Beyond the hype and hope: Critical considerations for intranasal oxytocin research in autism spectrum disorder. Autism Res. 10, 1-17 (2016).

13. Leng, G. \& Ludwig, M. Intranasal Oxytocin: Myths and Delusions. Biol. Psychiatry 79, 243-250 (2016).

14. Gwee, P.-C., Tay, B.-H., Brenner, S. \& Venkatesh, B. Characterization of the neurohypophysial hormone gene loci in elephant shark and the Japanese lamprey: origin of the vertebrate neurohypophysial hormone genes. BMC Evol. Biol. 9, 47 (2009).

15. Stafflinger, E. et al. Cloning and identification of an oxytocin/vasopressin-like receptor and its ligand from insects. Proc. Natl. Acad. Sci. 105, 3262-3267 (2008). 
16. Walum, H., Waldman, I. D. \& Young, L. J. Statistical and Methodological Considerations for the Interpretation of Intranasal Oxytocin Studies. Biol. Psychiatry 79, 251-257 (2016).

17. Muthukrishna, M. \& Henrich, J. A problem in theory. Nat. Hum. Behav. 3, 221229 (2019).

18. Klopfer, P. H. \& Klopfer, M. S. Maternal "imprinting" in goats: Fostering of alien young. Ethology 25, 862-866 (1968).

19. Kosfeld, M., Heinrichs, M., Zak, P. J., Fischbacher, U. \& Fehr, E. Oxytocin increases trust in humans. Nature 435, 673-676 (2005).

20. Nave, G., Camerer, C. \& McCullough, M. Does oxytocin increase trust in humans? A critical review of research. Perspect. Psychol. Sci. 10, 772-789 (2015).

21. Shamay-Tsoory, S. G. \& Abu-Akel, A. The social salience hypothesis of oxytocin. Biol. Psychiatry 79, 194-202 (2016).

22. Kemp, A. H. \& Guastella, A. J. The role of oxytocin in human affect a novel hypothesis. Curr. Dir. Psychol. Sci. 20, 222-231 (2011).

23. Quintana, D. S. \& Guastella, A. J. An allostatic theory of oxytocin. Trends Cogn. Sci. (2020) doi:https://doi.org/10.1016/j.tics.2020.03.008.

24. Harari-Dahan, O. \& Bernstein, A. A general approach-avoidance hypothesis of oxytocin: accounting for social and non-social effects of oxytocin. Neurosci. Biobehav. Rev. 47, 506-519 (2014). 
25. Quintana, D. S. et al. Low-dose intranasal oxytocin delivered with Breath Powered device modulates pupil diameter and amygdala activity: a randomized controlled pupillometry and fMRI study. Neuropsychopharmacology 44, 306-313 (2019).

26. Harari-Dahan, O. \& Bernstein, A. Oxytocin attenuates social and non-social avoidance: Re-thinking the social specificity of Oxytocin. Psychoneuroendocrinology 81, 105-112 (2017).

27. Fam, J., Holmes, N., Delaney, A., Crane, J. \& Westbrook, R. F. Oxytocin receptor activation in the basolateral complex of the amygdala enhances discrimination between discrete cues and promotes configural processing of cues. Psychoneuroendocrinology 96, 84-92 (2018).

28. Singer, T. et al. Effects of oxytocin and prosocial behavior on brain responses to direct and vicariously experienced pain. Emotion 8, 781-791 (2008).

29. Zhao, Z. et al. Oxytocin differentially modulates specific dorsal and ventral striatal functional connections with frontal and cerebellar regions. NeuroImage 184, 781-789 (2019).

30. Geerling, J. C., Shin, J. W., Chimenti, P. C. \& Loewy, A. D. Paraventricular hypothalamic nucleus: Axonal projections to the brainstem. J. Comp. Neurol. 518, 1460-1499 (2010). 
31. Browning, K. N. \& Travagli, R. A. Central nervous system control of gastrointestinal motility and secretion and modulation of gastrointestinal functions. Compr. Physiol. 4, 1339-1368 (2014).

32. Leng, G. \& Sabatier, N. Oxytocin - The Sweet Hormone? vol. 28 365-376 (2017).

33. Maejima, Y. et al. Peripheral oxytocin treatment ameliorates obesity by reducing food intake and visceral fat mass. Aging 3, 1169-77 (2011).

34. Sabatier, N., Leng, G. \& Menzies, J. Oxytocin, feeding, and satiety. Front. Endocrinol. 4, (2013).

35. Lawson, E. A. et al. Oxytocin reduces caloric intake in men. Obes. Silver Spring Md 23, 950-6 (2015).

36. Ott, V. et al. Oxytocin reduces reward-driven food intake in humans. Diabetes 62, 3418-3425 (2013).

37. Zhang, H. et al. Treatment of obesity and diabetes using oxytocin or analogs in patients and mouse models. PloS One 8, e61477 (2013).

38. Blevins, J. E. \& Baskin, D. G. Translational and therapeutic potential of oxytocin as an anti-obesity strategy: Insights from rodents, nonhuman primates and humans. Physiol. Behav. 152, 438-449 (2015).

39. Sclafani, A., Rinaman, L., Vollmer, R. R. \& Amico, J. A. Oxytocin knockout mice demonstrate enhanced intake of sweet and nonsweet carbohydrate solutions. Am. J. Physiol. Regul. Integr. Comp. Physiol. 292, R1828-33 (2007). 
40. Ho, J. M. \& Blevins, J. E. Coming full circle: contributions of central and peripheral oxytocin actions to energy balance. Endocrinology 154, 589-96 (2013).

41. Moos, F. et al. Release of oxytocin and vasopressin by magnocellular nuclei in vitro: specific facilitatory effect of oxytocin on its own release. J. Endocrinol. 102, 63-72 (1984).

42. Neumann, I., Koehler, E., Landgraf, R. \& Summy-Long, J. An oxytocin receptor antagonist infused into the supraoptic nucleus attenuates intranuclear and peripheral release of oxytocin during suckling in conscious rats. Endocrinology 134, 141-8 (1994).

43. Blevins, J. E. et al. Chronic oxytocin administration inhibits food intake, increases energy expenditure, and produces weight loss in fructose-fed obese rhesus monkeys. Am. J. Physiol. Regul. Integr. Comp. Physiol. 308, R431-8 (2015).

44. Insel, T. R. Translating Oxytocin Neuroscience to the Clinic: A National Institute of Mental Health Perspective. Biol. Psychiatry 79, 153-154 (2016).

45. King, L. B., Walum, H., Inoue, K., Eyrich, N. W. \& Young, L. J. Variation in the Oxytocin Receptor Gene Predicts Brain Region-Specific Expression and Social Attachment. Biol. Psychiatry (2015).

46. Keebaugh, A. C. \& Young, L. J. Increasing oxytocin receptor expression in the nucleus accumbens of pre-pubertal female prairie voles enhances alloparental responsiveness and partner preference formation as adults. Horm. Behav. 60, 498$504(2011)$. 
47. Loup, F., Tribollet, E., Dubois-Dauphin, M. \& Dreifuss, J. J. Localization of highaffinity binding sites for oxytocin and vasopressin in the human brain. An autoradiographic study. Brain Res. 555, 220-232 (1991).

48. Uhrig, S. et al. Reduced oxytocin receptor gene expression and binding sites in different brain regions in schizophrenia: A post-mortem study. Schizophr. Res. (2016).

49. Yarkoni, T., Poldrack, R. A., Nichols, T. E., Van Essen, D. C. \& Wager, T. D. Large-scale automated synthesis of human functional neuroimaging data. Nat. Methods 8, 665 (2011).

50. Quintana, D. S. et al. Oxytocin pathway gene networks in the human brain. Nat. Commun. 10, 668 (2019).

51. Paloyelis, Y. et al. A Spatiotemporal Profile of In Vivo Cerebral Blood Flow Changes Following Intranasal Oxytocin in Humans. Biol. Psychiatry (2014).

52. Beard, R., Singh, N., Grundschober, C., Gee, A. D. \& Tate, E. W. High-yielding $18 \mathrm{~F}$ radiosynthesis of a novel oxytocin receptor tracer, a probe for nose-to-brain oxytocin uptake in vivo. Chem. Commun. 54, 8120-8123 (2018).

53. Love, T. M. Oxytocin, motivation and the role of dopamine. Pharmacol. Biochem. Behav. 119, 49-60 (2014).

54. Feldman, R., Feldman, R., Monakhov, M., Pratt, M. \& Ebstein, R. P. Oxytocin Pathway Genes : Evolutionary Ancient System Impacting on Human Affiliation , Review Oxytocin Pathway Genes : Evolutionary Ancient System Impacting on 
Human Af fi liation ,. Biol. Psychiatry 1-11 (2015)

doi:10.1016/j.biopsych.2015.08.008.

55. Gimpl, G., Farenholtz, F., Fahrenholz, F. \& Gene, C. The oxytocin receptor system: Structure, function and regulation. Physiol. Rev. 81, 629-683 (2001).

56. Colaianni, G. et al. Regulated production of the pituitary hormone oxytocin from murine and human osteoblasts. Biochem. Biophys. Res. Commun. 411, 512-515 (2011).

57. Tamma, R. et al. Oxytocin is an anabolic bone hormone. Proc. Natl. Acad. Sci. U. S. A. 106, 7149-7154 (2009).

58. Stanley, S. A. et al. Bidirectional electromagnetic control of the hypothalamus regulates feeding and metabolism. Nature 531, 647-650 (2016).

59. Noble, E. E., Billington, C. J., Kotz, C. M., Wang, C. \& Wang, C. F. Oxytocin in the ventromedial hypothalamic nucleus reduces feeding and acutely increases energy expenditure. Am J Physiol Regul Integr Comp Physiol 307, 737-745 (2014).

60. Jankowski, M. et al. Oxytocin and its receptors are synthesized in the rat vasculature. Proc. Natl. Acad. Sci. U. S. A. 97, 6207-6211 (2000).

61. Norman, G. J. et al. Oxytocin increases autonomic cardiac control: Moderation by loneliness. Biol. Psychol. 86, 174-180 (2011). 
62. Gutkowska, J., Jankowski, M., Mukaddam-Daher, S. \& McCann, S. M. Oxytocin is a cardiovascular hormone. Braz. J. Med. Biol. Res. Rev. Bras. Pesqui. Medicas E Biol. 33, 625-33 (2000).

63. Gutkowska, J. et al. Oxytocin releases atrial natriuretic peptide by combining with oxytocin receptors in the heart. Proc. Natl. Acad. Sci. U. S. A. 94, 1170411709 (1997).

64. Yang, H.-P., Wang, L., Han, L. \& Wang, S. C. Nonsocial Functions of Hypothalamic Oxytocin. ISRN Neurosci. 2013, (2013).

65. Lumsden, N. G. \& Hobbs, R. S. K. and A. J. C-type Natriuretic Peptide (CNP): Cardiovascular Roles and Potential as a Therapeutic Target. Current Pharmaceutical Design http://www.eurekaselect.com/73288/article (2010).

66. Palkovits, M. Interconnections between the neuroendocrine hypothalamus and the central autonomic system. Geoffrey Harris Memorial Lecture, Kitakyushu, Japan, October 1998. Front. Neuroendocrinol. 20, 270-295 (1999).

67. Swanson, L. W. \& Kuypers, H. G. J. M. The paraventricular nucleus of the hypothalamus: Cytoarchitectonic subdivisions and organization of projections to the pituitary, dorsal vagal complex, and spinal cord as demonstrated by retrograde fluorescence double-labeling methods. J. Comp. Neurol. 194, 555-570 (1980). 
68. Valentino, R. J., Foote, S. L. \& Page, M. E. The Locus Coeruleus as a Site for Integrating Corticotropin-Releasing Factor and Noradrenergic Mediation of Stress Responsesa. Ann. N. Y. Acad. Sci. 697, 173-188 (1993).

69. Grewen, K. M. \& Light, K. C. Plasma oxytocin is related to lower cardiovascular and sympathetic reactivity to stress. Biol. Psychol. 87, 340-349 (2011).

70. Tops, M., Peer, J. M. V., Korf, J., Wijers, A. A. \& Tucker, D. M. Anxiety, cortisol, and attachment predict plasma oxytocin. Psychophysiology 44, 444-449 (2007)

71. Nicolson, N. A., Davis, M. C., Kruszewski, D. \& Zautra, A. J. Childhood Maltreatment and Diurnal Cortisol Patterns in Women With Chronic Pain. Psychosom. Med. 72, 471-480 (2010).

72. Gutkowska, J. \& Jankowski, M. Oxytocin Revisited: Its Role in Cardiovascular Regulation. J. Neuroendocrinol. 24, 599-608 (2012).

73. Quintana, D. S., Kemp, A. H., Alvares, G. A. \& Guastella, A. J. A role for autonomic cardiac control in the effects of oxytocin on social behavior and psychiatric illness. Front. Neurosci. 7, (2013).

74. Porges, S. W. The Polyvagal Theory: Neurophysiological Foundations of Emotions, Attachment, Communication, and Self-regulation. (W. W. Norton \& Company, 2011). 
75. Akselrod, S. et al. Power spectrum analysis of heart rate fluctuation: a quantitative probe of beat-to-beat cardiovascular control. Science $\mathbf{2 1 3}, 220-222$ (1981).

76. Quintana, D. S., Guastella, A. J., Outhred, T., Hickie, I. B. \& Kemp, A. H. Heart rate variability is associated with emotion recognition: Direct evidence for a relationship between the autonomic nervous system and social cognition. Int. J. Psychophysiol. 86, 168-172 (2012).

77. Butler, E. A., Wilhelm, F. H. \& Gross, J. J. Respiratory sinus arrhythmia, emotion, and emotion regulation during social interaction. Psychophysiology 43, 612-622 (2006).

78. Berntson, G. G., Cacioppo, J. T. \& Grossman, P. Whither vagal tone. Biol. Psychol. 74, 295-300 (2007).

79. Kemp, A. H. et al. Oxytocin Increases Heart Rate Variability in Humans at Rest: Implications for Social Approach-Related Motivation and Capacity for Social Engagement. PLoS ONE 7, e44014 (2012).

80. Quintana, D. S. et al. Low dose oxytocin delivered intranasally with Breath Powered device affects social-cognitive behavior: a randomized 4-way crossover trial with nasal cavity dimension assessment. Transl. Psychiatry 5, e602 (2015).

81. Benarroch, E. E. The central autonomic network: functional organization, dysfunction, and perspective. Mayo Clin. Proc. 68, 988-1001 (1993). 
82. Quintana, D. S. \& Guastella, A. An Allostatic Theory of Oxytocin.

https://osf.io/j7tnf (2019) doi:10.31219/osf.io/j7tnf.

83. Li, Y., Field, P. M. \& Raisman, G. Olfactory ensheathing cells and olfactory nerve fibroblasts maintain continuous open channels for regrowth of olfactory nerve fibres. Glia 52, 245-251 (2005).

84. Thorne, R. G., Emory, C. R., Ala, T. A. \& Frey II, W. H. Quantitative analysis of the olfactory pathway for drug delivery to the brain. Brain Res. 692, 278-282 (1995).

85. Guastella, A. J. et al. Recommendations for the standardisation of oxytocin nasal administration and guidelines for its reporting in human research. Psychoneuroendocrinology 38, 612-625 (2013).

86. Gizurarson, S. Anatomical and Histological Factors Affecting Intranasal Drug and Vaccine Delivery. Curr. Drug Deliv. 9, 566-582 (2012).

87. Smith, A. S., Korgan, A. C. \& Young, W. S. Oxytocin delivered nasally or intraperitoneally reaches the brain and plasma of normal and oxytocin knockout mice. Pharmacol. Res. 104324 (2019) doi:10.1016/j.phrs.2019.104324.

88. Striepens, N. et al. Elevated cerebrospinal fluid and blood concentrations of oxytocin following its intranasal administration in humans. Sci. Rep. 3, (2013).

89. Dal Monte, O., Noble, P. L., Turchi, J., Cummins, A. \& Averbeck, B. B. CSF and Blood Oxytocin Concentration Changes following Intranasal Delivery in Macaque. PLoS ONE 9, e103677 (2014). 
90. Lee, M. R. et al. Oxytocin by intranasal and intravenous routes reaches the cerebrospinal fluid in rhesus macaques: determination using a novel oxytocin assay. Mol. Psychiatry (2017).

91. Hollander, E. et al. Oxytocin Increases Retention of Social Cognition in Autism. Biol. Psychiatry 61, 498-503 (2007).

92. Hollander, E. et al. Oxytocin Infusion Reduces Repetitive Behaviors in Adults with Autistic and Asperger's Disorders. Neuropsychopharmacology 28, 193-198 (2003).

93. Neumann, I. D., Maloumby, R., Beiderbeck, D. I., Lukas, M. \& Landgraf, R. Increased brain and plasma oxytocin after nasal and peripheral administration in rats and mice. Psychoneuroendocrinology 38, 1985-1993 (2013).

94. Quintana, D. S. et al. Low dose intranasal oxytocin delivered with Breath Powered device dampens amygdala response to emotional stimuli: A peripheral effect-controlled within-subjects randomized dose-response fMRI trial. Psychoneuroendocrinology 69, 180-188 (2016).

95. Quintana, D. S. et al. Low-dose intranasal oxytocin delivered with Breath Powered device modulates pupil diameter and amygdala activity: a randomized controlled pupillometry and fMRI study. Neuropsychopharmacology 1 (2018).

96. Quintana, D. S., Guastella, A. J., Westlye, L. T. \& Andreassen, O. A. The promise and pitfalls of intranasally administering psychopharmacological agents for the treatment of psychiatric disorders. Mol. Psychiatry 21, 29-38 (2016). 
97. Valstad, M. et al. The correlation between central and peripheral oxytocin concentrations: A systematic review and meta-analysis. Neurosci. Biobehav. Rev. 78, 117-124 (2017).

98. Baker, M. 1,500 scientists lift the lid on reproducibility. Nat. News 533, 452 (2016).

99. Munafò, M. R. et al. A manifesto for reproducible science. Nat. Hum. Behav. 1, 19 (2017).

100. Morey, R. \& Lakens, D. Why most of psychology is statistically unfalsifiable. (2016) doi:10.5281/zenodo.838685.

101. Dienes, Z. Using Bayes to get the most out of non-significant results. Front. Psychol. 5, (2014).

102. Hauck, W. W. \& Anderson, S. A new statistical procedure for testing equivalence in two-group comparative bioavailability trials. J. Pharmacokinet. Biopharm. 12, 83-91 (1984).

103. Lakens, D. Equivalence Tests: A Practical Primer for t Tests, Correlations, and Meta-Analyses. Soc. Psychol. Personal. Sci. 8, 355-362 (2017).

104. Wagenmakers, E.-J. et al. Bayesian inference for psychology. Part I: Theoretical advantages and practical ramifications. Psychon. Bull. Rev. 25, 35-57 (2018).

105. Quintana, D. S. \& Williams, D. R. Bayesian alternatives for common nullhypothesis significance tests in psychiatry: a non-technical guide using JASP. BMC Psychiatry 2018181 18, 178 (2018). 
106. Quintana, D. S. Revisiting non-significant effects of intranasal oxytocin using equivalence testing. Psychoneuroendocrinology 87, 127-130 (2018).

107. Tabak, B. A. et al. Null results of oxytocin and vasopressin administration across a range of social cognitive and behavioral paradigms: Evidence from a randomized controlled trial. Psychoneuroendocrinology 107, 124-132 (2019).

108. Reiter, J. P. Releasing multiply imputed, synthetic public use microdata: an illustration and empirical study. J. R. Stat. Soc. Ser. A Stat. Soc. 168, 185-205 (2005).

109. Quintana, D. S. A synthetic dataset primer for the biobehavioural sciences to promote reproducibility and hypothesis generation. eLife 9, e53275 (2020).

110. Lane, A., Luminet, O., Nave, G. \& Mikolajczak, M. Is there a publication bias in behavioral intranasal oxytocin research on humans? Opening the file drawer of one lab. J. Neuroendocrinol. 28, (2016).

111. McCullough, M. E., Churchland, P. S. \& Mendez, A. J. Problems with measuring peripheral oxytocin: can the data on oxytocin and human behavior be trusted? Neurosci. Biobehav. Rev. 37, 1485-1492 (2013).

112. Marín, O. Developmental timing and critical windows for the treatment of psychiatric disorders. Nat. Med. 22, 1229-1238 (2016).

113. Kamesh, N., Aradhyam, G. K. \& Manoj, N. The repertoire of G protein-coupled receptors in the sea squirt Ciona intestinalis. BMC Evol. Biol. 8, 129 (2008). 
114. Quintana, D. S. Genetic networks of the oxytocin system in the human brain: A gene expression and large-scale fMRI meta-analysis study Daniel S. Quintana. (2017).

115. Ding, C., Leow, M. K. -S. \& Magkos, F. Oxytocin in metabolic homeostasis: implications for obesity and diabetes management. Obes. Rev. 20, 22-40 (2019).

116. Nesse, R. M. Tinbergen's four questions: Two proximate, two evolutionary. Evol. Med. Public Health 2019, 2-2 (2019).

117. Fujino, Y. et al. Possible functions of oxytocin/vasopressin-superfamily peptides in annelids with special reference to reproduction and osmoregulation. J. Exp. Zool. 284, 401-406 (1999).

118. Kawada, T., Sekiguchi, T., Itoh, Y., Ogasawara, M. \& Satake, H. Characterization of a novel vasopressin/oxytocin superfamily peptide and its receptor from an ascidian, Ciona intestinalis. Peptides 29, 1672-1678 (2008).

119. Quintana, D. \& Guastella, A. An integrative allostatic account of oxytocin: maintaining stability through change. https://osf.io/j7tnf (2019) doi:10.31219/osf.io/j7tnf.

120. Beets, I. et al. Vasopressin/oxytocin-related signaling regulates gustatory associative learning in C. elegans. Science 338, 543-545 (2012).

121. Chini, B., Leonzino, M., Braida, D. \& Sala, M. Learning about oxytocin: pharmacologic and behavioral issues. Biol. Psychiatry 76, 360-366 (2014). 
122. Wallis, M. Molecular evolution of the neurohypophysial hormone precursors in mammals: Comparative genomics reveals novel mammalian oxytocin and vasopressin analogues. Gen. Comp. Endocrinol. 179, 313-318 (2012).

123. Freeman, S. M. \& Young, L. J. Comparative Perspectives on Oxytocin and Vasopressin Receptor Research in Rodents and Primates: Translational Implications. J. Neuroendocrinol. 28, (2016).

124. Carter, C. S. Sex differences in oxytocin and vasopressin: Implications for autism spectrum disorders? Behav. Brain Res. 176, 170-186 (2007).

125. Holt-Lunstad, J., Birmingham, W. \& Light, K. C. The influence of depressive symptomatology and perceived stress on plasma and salivary oxytocin before, during and after a support enhancement intervention. Psychoneuroendocrinology 36, 1249-1256 (2011).

126. Weisman, O., Zagoory-Sharon, O., Schneiderman, I., Gordon, I. \& Feldman, R. Plasma oxytocin distributions in a large cohort of women and men and their gender-specific associations with anxiety. Psychoneuroendocrinology 38, 694-701 (2013).

127. Zhong, S. et al. U-Shaped Relation between Plasma Oxytocin Levels and Behavior in the Trust Game. PLOS ONE 7, e51095 (2012).

128. Lefevre, A. et al. A comparison of methods to measure central and peripheral oxytocin concentrations in human and non-human primates. Sci. Rep. 7, 1-10 (2017). 
129. Kirsch, P. et al. Oxytocin Modulates Neural Circuitry for Social Cognition and Fear in Humans. J. Neurosci. 25, 11489-11493 (2005).

130. Lischke, A. et al. Oxytocin increases amygdala reactivity to threatening scenes in females. Psychoneuroendocrinology 37, 1431-1438 (2012).

131. Frijling, J. L. Preventing PTSD with oxytocin: effects of oxytocin administration on fear neurocircuitry and PTSD symptom development in recently traumaexposed individuals. Eur. J. Psychotraumatology 8, (2017).

132. Geng, Y. et al. Oxytocin Enhancement of Emotional Empathy: Generalization Across Cultures and Effects on Amygdala Activity. Front. Neurosci. 12, (2018).

133. Feldman, R. et al. Sensitive Parenting Is Associated with Plasma Oxytocin and Polymorphisms in the OXTR and CD38 Genes. Biol. Psychiatry 72, 175-181 (2012).

134. Rilling, J. K. et al. Sex differences in the neural and behavioral response to intranasal oxytocin and vasopressin during human social interaction. Psychoneuroendocrinology 39, (2014).

135. Lieberz, J. et al. Kinetics of oxytocin effects on amygdala and striatal reactivity vary between women and men. Neuropsychopharmacology 1-7 (2019) doi:10.1038/s41386-019-0582-6.

136. Simonsohn, U., Nelson, L. D. \& Simmons, J. P. p-Curve and Effect Size: Correcting for Publication Bias Using Only Significant Results. Perspect. Psychol. Sci. 9, 666-681 (2014). 
137. Gilbert, C., Brown, M. C. J., Cappelleri, J. C., Carlsson, M. \& McKenna, S. P. Estimating a Minimally Important Difference in Pulmonary Arterial Hypertension Following Treatment With Sildenafil. Chest 135, 137-142 (2009).

138. Lakens, D., Scheel, A. M. \& Isager, P. M. Equivalence Testing for Psychological Research: A Tutorial.

139. Faul, F., Erdfelder, E., Lang, A.-G. \& Buchner, A. G*Power 3: A flexible statistical power analysis program for the social, behavioral, and biomedical sciences. Behav. Res. Methods 39, 175-191 (2007).

140. DeBruine, L. \& Barr, D. J. Understanding mixed effects models through data simulation. (2019).

141. Grinevich, V., Desarménien, M. G., Chini, B., Tauber, M. \& Muscatelli, F. Ontogenesis of oxytocin pathways in the mammalian brain: late maturation and psychosocial disorders. Front. Neuroanat. 8, (2015).

142. Goudsmit, E., Neijmeijer-Leloux, A. \& Swaab, D. F. The human hypothalamoneurohypophyseal system in relation to development, aging and Alzheimer's disease. Prog. Brain Res. 93, 237-247; discussion 247-248 (1992).

143. Hammock, E. A. D. Developmental perspectives on oxytocin and vasopressin. Neuropsychopharmacol. Off. Publ. Am. Coll. Neuropsychopharmacol. 40, 24-42 (2015).

144. Vaidyanathan, R. \& Hammock, E. A. D. Oxytocin receptor dynamics in the brain across development and species. Dev. Neurobiol. 77, 143-157 (2017). 
145. Zheng, J.-J. et al. Oxytocin mediates early experience-dependent cross-modal plasticity in the sensory cortices. Nat. Neurosci. 17, 391-399 (2014).

146. Winslow, J. T. et al. Infant vocalization, adult aggression, and fear behavior of an oxytocin null mutant mouse. Horm. Behav. 37, 145-155 (2000).

147. Alberts, J. R. Huddling by rat pups: ontogeny of individual and group behavior. Dev. Psychobiol. 49, 22-32 (2007).

148. Feldman, R. Oxytocin and social affiliation in humans. Horm. Behav. 61, 380-391 (2012).

149. Clark, C. L. et al. Neonatal CSF oxytocin levels are associated with parent report of infant soothability and sociability. Psychoneuroendocrinology 38, 1208-1212 (2013).

150. Quintana, D. S., Dieset, I., Elvsåshagen, T., Westlye, L. T. \& Andreassen, O. A. Oxytocin system dysfunction as a common mechanism underlying metabolic syndrome and psychiatric symptoms in schizophrenia and bipolar disorders. Front. Neuroendocrinol. 45, 1-10 (2017). 